\title{
Magnetic-Particle-Imaging mit mehreren Gradientenstärken
}

\author{
Patryk Szwargulski ${ }^{1,2}$, Nadine Gdaniec ${ }^{1,2}$, Tobias Knopp ${ }^{1,2}$ \\ ${ }^{1}$ Abteilung für Biomedizinische Bildgebung, Universitätsklinikum \\ Hamburg-Eppendorf \\ ${ }^{2}$ Institut für Biomedizinische Bildgebung, Technische Universität Hamburg \\ p.szwargulski@uke.de
}

Die Magnetpartikelbildgebung (engl. Magnetic-Particle-Imaging, MPI) ist ein tomografisches Bildgebungsverfahren mit dem super-paramagnetische Nanopartikel mit einer hohen zeitlichen Auflösung visualisiert werden können [1]. Die räumliche Auflösung und die Größe des Bildgebungsbereiches hängen direkt mit der genutzten Gradientenfeldstärke zusammen. Bei einer hohen Gradientenfeldstärke kann zwar eine sehr gute räumliche Auflösung erreicht werden, gleichzeitig verringert sich allerdings der Bildgebungsbereich. Um ein größeres Volumen mit einer hohen räumlichen Aufösung vermessen zu können werden multi-patch Ansätze verwendet bei denen der Bildgebungsbereich mithilfe weiterer Felder im Raum verschoben wird [2]. Da die gesamte Messzeit mit der Anzahl der zu messenden Patches linear zunimmt wird die zeitliche Auflösung dabei aber reduziert. In dieser Arbeit wird eine Methode vorgestellt, bei der zuerst ein schneller niedrig aufgelöster Übersichtsscann aufgenommen wird. Dieser wird anschließend dazu genutzt efine geringe Anzahl von hoch aufgelösten Messungen zu planen. Dabei werden nur solche Bereiche abgetastet die überhaupt Magnetpartikel enthalten. Gerade bei angiographischen Messungen kann so ein großes Volumen mit einer anisotropen räumlichen Aufösung in einem Bruchteil der Zeit aufgenommen werden, die bei der konventionellen Methode notwendig wäre. Die Rohdaten der verschiedenen Messungen werden dazu in einem gemeinsamen linearen Gleichungssystem zusammengefasst und anschließend mittels iterativer Verfahren gelöst [3].

\section{Literaturverzeichnis}

1. Gleich B, Weizenecker J. Tomographic imaging using the nonlinear response of magnetic particles. Nature. 2005;435(7046):1214 - 1217.

2. Rahmer J, Gleich B, Bontus C, et al. Rapid 3D in vivo magnetic particle imaging with a large field of view. Proc Int Soc Magn Reson Med. 2011;19:3285.

3. Gdaniec N, Szwargulski P, Knopp T. Fast multiresolution data acquisition for magnetic particle imaging using adaptive feature detection. J Med Phys. 2017;44(12):6456-6460. Available from: http://dx.doi.org/10.1002/mp.12628. 